\title{
Dietary Intervention with Oatmeal in Patients with uncontrolled Type 2 Diabetes Mellitus - A Crossover Study
}

\section{Authors}

Graciela Delgado $^{1}$ *, Marcus E. Kleber ${ }^{\text {* }}$, Bernhard K. Krämer ${ }^{1}$, Michael Morcos ${ }^{2}$, Per M. Humpert ${ }^{2}$, Kerstin Wiegand ${ }^{1}$, Andy Mauldin', Klaus Kusterer ${ }^{3}$, Michael Enghöfer ${ }^{3}$, Winfried März', 4, 5, Thomas Segiet ${ }^{6 \#, ~ A l e x a n d e r ~ L a m m e r t ~}{ }^{1,7 \#}$

\section{Affiliations}

1 Fifth Department of Medicine, Medical Faculty Mannheim, Heidelberg University, Germany

2 Stoffwechselzentrum Rhein-Pfalz, Belchenstrasse 1-5, Mannheim, Germany

3 Endokrinologische Gemeinschaftpraxis Mannheim, Kurfürstenpassage P7 24, Mannheim

4 Clinical Institute of Medical and Chemical Laboratory Diagnostics, Medical University Graz, Graz, Austria

5 Synlab Academy, Synlab Holding Deutschland GmbH, Mannheim, Germany

6 Diabetologische Schwerpunktpraxis Dres. Segiet und Bode, Speyer, Germany

7 Praxis für Diabetes, Stoffwechsel-und Nierenerkrankungen, Verladeplatz 2-4, Grünstadt, Germany

Key words

insulin dose, fasting glucose, glycemic control

received 24.05 .2018

revised 31.07 .2018

accepted 08.08.2018

Bibliography

DOI https://doi.org/10.1055/a-0677-6068

Published online: 29.8.2018

Exp Clin Endocrinol Diabetes 2019; 127: 623-629

(c) J. A. Barth Verlag in Georg Thieme Verlag KG Stuttgart .

New York

ISSN 0947-7349

\section{Correspondence}

Priv.-Doz. Dr. med. Alexander Lammert

Praxis für Diabetes, Stoffwechsel-und Nierenerkrankungen

Grünstadt

Verladeplatz 2-4

67269 Grünstadt

Germany

dr.alex.lammert@gmail.com
Supporting Information for this article is available online at https://doi.org/10.1055/a-0677-6068

\begin{abstract}
Background In a pilot study, we evaluated the efficacy of two days of oatmeal on insulin resistance and glucose metabolism and found a marked decrease of insulin requirements. The most important shortcoming of that study was that the interventions were not isocaloric (diabetes adapted diet: $1500 \mathrm{kcal} / \mathrm{d}$ vs. oatmeal $1100 \mathrm{kcal} / \mathrm{d}$ ). To address these drawbacks we designed the OatMeal And Insulin Resistance (OMA-IR) study. Methods The study was a randomized, open label crossover dietary intervention study with consecutive inclusion of 15 patients with uncontrolled type 2 diabetes. The intervention comprised two days of oatmeal on days 3 and 4 of a 5 days hospital stay. During the control period, patients received a diabetes mellitus adapted diet only. The primary endpoint was the daily insulin requirement and glycemic control.

Results Upon oatmeal treatment, the required insulin dose could be significantly reduced on the third and fourth day as compared to the second day of inpatient stay $(82.0 \pm 30.3$ and $69.9 \pm 29$.9IU versus $112 \pm 36.2 \mathrm{IU} ; \mathrm{P}<0.001)$. During control treatment, insulin requirement did not change. There were no significant differences in the changes of mean blood glucose or fasting glucose between both treatments. HbA1c was lower four weeks after the oatmeal intervention.

Conclusion In this crossover study, two days of oatmeal intervention allowed a highly significant reduction of required daily insulin doses while maintaining adequate metabolic control as compared to a diabetes adapted diet only. The beneficial effects of the intervention might last for several weeks as shown by the lower HbA1c four weeks after the intervention.
\end{abstract}




\section{Introduction}

In 1903, Carl von Noorden reported the treatment of diabetic patients with an oatmeal diet without fat restriction, which diminished glucosuria and ketonuria [1]. Despite successes in diabetes treatment using such diets, the so-called "starvation diet" with extreme calorie restriction advocated by Allen in 1915 [2] dominated diabetes treatment until the introduction of insulin. Allen's diet increased the life expectancy of insulin-dependent patients but it was hardly accepted by the patients and some of them even starved to death [3]. Nowadays, treatment of type 2 diabetes mellitus (T2DM) is complicated by the increasing prevalence of obesity and insulin resistance. Especially in patients with uncontrolled T2DM, management of blood glucose may be severely hampered due to exorbitant insulin dosages with a minor risk of hypoglycaemia but a major one for weight gain, causing an aggravation of insulin resistance.

Both, a diet rich in certain carbohydrates, fibre, particularly beta-glucans, as well as caloric restriction are still practiced in patients with uncontrolled diabetes mellitus and severe insulin resistance today. In clinical practice, these patients are treated for several days with these diets to reduce insulin requirements and to improve glycemia. According to this type of intervention, these days are the so called "Kohlenhydrattage" which translates into carbohydrate days. In Germany, we traditionally used oat as standardized carbohydrate source. It resembles the intervention of Carl von Noorden except that it is not enhanced with butter and eggs to allow for caloric restriction. James B. Herrick already stated in his case series 1908 that this intervention is effective, while occasionally the stomach will rebel and refuse to tolerate this food for any great length of time [4] Hence, many dietitians have modulated this intervention with more complex or tasty dietary advice with the emphasis on caloric restriction and carbohydrate dominance, which is mainly achieved by adding vegetables to rice, bread, potatoes and other sources of carbohydrates. Furthermore, these diets are/were a mainstay within the in-hospital management of patients with uncontrolled diabetes $[5,6]$. Even though they are regarded as valuable clinical tools, there is no good evidence of their efficiency.

Oats are rich in beta-glucan and therefore represent a good source of soluble dietary fibre. The European Food Safety Agency (EFSA) has examined several claims related to health benefits through the consumption of beta-glucans from oats and barley and approved their effect for: (i) maintenance of normal blood LDL-cholesterol concentrations and (ii) reduction of post-prandial glycaemic responses [7]. For diabetic patients it is usually recommended to consume at least an equal amount of fibres and whole grains as it is recommended for the general population (about $14 \mathrm{~g}$ fibre/1000 kcals daily). Dietary fibres are thought to promote beneficial effects such as laxation, reduction in blood lipids or modulation of blood glucose due to their non-digestibility in the small intestine and fermentation in the colon.

A recent systematic review and meta-analysis investigated whether oats intake is beneficial for patients with diabetes mellitus and concluded that ingestion of oats and or oat related fibre significantly reduces the acute postprandial glucose and insulin responses compared with different non-standardised control diets [8]. Oat has a high content of fibres that might act in different ways to ameliorate glucose metabolism (reviewed in [9]).
In a previous pilot study, we evaluated the efficacy of two days of oatmeal on insulin resistance and glucose metabolism in a clinical setting. We found a marked decrease of insulin requirements ( $40 \%)$ and mean daily blood glucose to a mean blood glucose of $114.7 \pm 36.7 \mathrm{mg} / \mathrm{dl}$. The effect persisted after a four-week outpatient period [10]. The most important limitation of this study was the two-step caloric restriction within the hospital stay (diabetesadapted diet: $1500 \mathrm{kcal} / \mathrm{d}$ vs. oatmeal $1100 \mathrm{kcal} / \mathrm{d}$ ) making it difficult to attribute the observed effects to oatmeal alone. To address these drawbacks, we designed an open label, crossover study with isocaloric interventions of oatmeal vs. hospital diet, the OatMeal And Insulin Resistance (OMA-IR) study. We hypothesized that a two-day intervention with oatmeal leads to a stronger amelioration of glucose control and a greater reduction of the insulin dosage as compared to standard DM-adapted diet.

\section{Material and Methods}

The study was performed as a randomized, open label crossover dietary intervention study with consecutive inclusion of 15 patients in an inpatient clinical setting. Our aim was to compare a dietary intervention consisting of two days of oatmeal with a conventional DM-adapted diet in patients with severe DM. Patients were hospitalized for uncontrolled diabetes mellitus and started on a DMadapted diet ( $1200 \mathrm{kcal}$ per day) for 2 days. The diabetes-adapted diet had a macronutrient content of 50-55\% carbohydrates, 15 $20 \%$ protein and $30 \%$ fat with $\sim 20 \mathrm{~g}$ of fibre daily. The intervention comprised two days of oatmeal (third and fourth day) within a 5 day hospital stay. Oatmeal was cooked with water. Cinnamon and herbs were the only extra ingredients. Every meal contained $100 \mathrm{~g}$ of oat resulting in an overall energy content of 1100 to $1200 \mathrm{kcal}$ per day with a macronutrient content of $63 \%$ carbohydrates $12 \%$ protein, $7 \%$ fat and $16.2 \mathrm{~g}$ of fibre ( $30 \%$ soluble and $70 \%$ insoluble).During the control period, patients received the DM-adapted diet $(1200 \mathrm{kcal}$ per day) only for five days. Thereafter, the patients were followed every four weeks for an overall of at least 12 weeks ( $\mathbf{F i g}$. 1). The washout phase between both visits was at least 3 up to 10 months (mean 5.5 months). Randomization was done by flipping a coin.

Inclusion criteria for the study were: type-2 diabetes mellitus (T2DM), inappropriate glucose control (HbA1c $>8 \%$ ), insulin dependency and insulin-resistance defined as a daily insulin dose of more than one unit per kilogram body weight. Exclusion criteria were any acute vascular event within the last 3 months, planned weight reducing therapy, acute and chronic inflammatory disease, therapy with corticosteroids and pregnancy.

Primary outcomes were daily insulin requirements and glycaemic control directly before and after intervention. Secondary outcome measures were changes in factors related to insulin resistance, changes in markers of inflammation and macrovascular risk like C-reactive protein, cholesterol, triglycerides, HDL cholesterol and LDL cholesterol directly before and after intervention.

The study was approved by the local ethics committee and registered on ClinicalTrials.gov (NCT00401453). All patients gave their written informed consent for participation in the study.

At each hospital stay, the insulin-dose was adjusted in a two-day run in phase to achieve adequate glycemic control. During this phase, all patients received a DM-adapted diet. Thereafter, the 


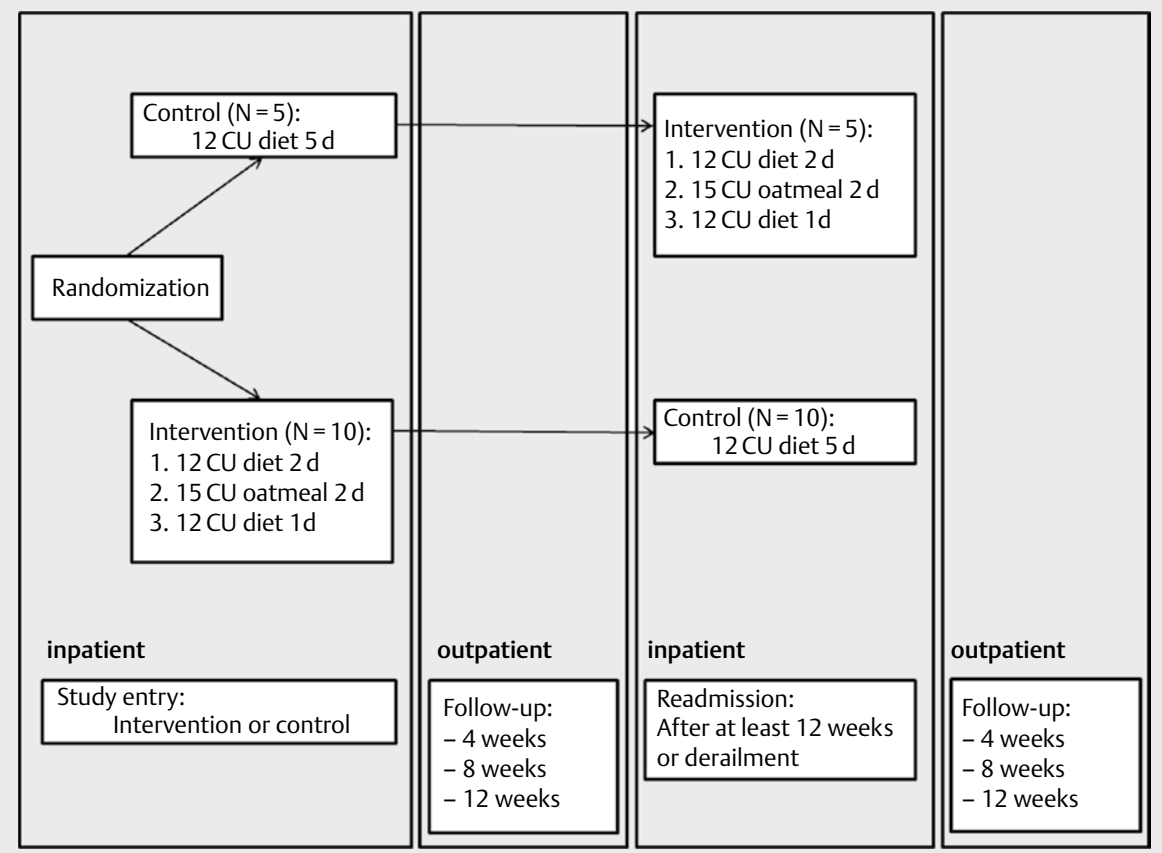

- Fig. 1 Flow chart of the study design; CU: Carbohydrate Unit.

- Table 1 Study characteristics at baseline.

\begin{tabular}{|l|c|c|}
\hline & $\begin{array}{c}\text { Mean (SD) or median } \\
\text { (25th to 75th } \\
\text { percentile) }\end{array}$ & Range \\
\hline Age (years) & $58.6(10.1)$ & $41-72$ \\
\hline Male sex (\%) & 53.3 & $31-42$ \\
\hline BMI (kg/m²) & $36.0(3.7)$ & $104-340$ \\
\hline Total cholesterol (mg/dl) & $215(63.7)$ & $59-214$ \\
\hline LDL-C (mg/dl) & $127(47.6)$ & $25-68$ \\
\hline HDL-C (mg/dl) & $40.5(12.6)$ & $73-654$ \\
\hline Triglycerides (\%) & $283(154)$ & $79-312$ \\
\hline Fasting glucose (mg/dl) & $136(100-203)$ & $75-195$ \\
\hline Total daily insulin dose & $103(77-134)$ & $48-140$ \\
\hline eGFR (ml/min/1.73 m²) & $76.7(25.4)$ & $3-45$ \\
\hline Diabetes duration (years) & $10(8.75-24.3)$ & \\
\hline Hypertension (\%) & 80.0 & \\
\hline Coronary heart disease (\%) & 26.7 & \\
\hline Peripheral artery disease (\%) & 13.3 & \\
\hline Diabetic retinopathy (\%) & 60.0 & \\
\hline Diabetic Nephropathy (\%) & 20.0 & \\
\hline Diabetic neuropathy (\%) & 60.0 & \\
\hline Metformin (\%) & 53.3 & \\
\hline Statin (\%) & 40 & \\
\hline $\begin{array}{l}\text { ACE inhibitor / AT2 receptor } \\
\text { antagonist (\%) }\end{array}$ & 66.7 & \\
\hline Acetyl salicylic acid (\%) & 53.3 & \\
\hline
\end{tabular}

dietary intervention was performed for 2 days with oatmeal during the oatmeal phase whereas the normal DM-adapted diet was continued during the control phase. Both diets contained between 1100 to $1200 \mathrm{kcal}$ per day. Within the oatmeal phase, the insulin dosage was reduced to prevent hypoglycaemia. The intervention was completed with one day of normal diabetes mellitus-adapted diet for final titration of the insulin dosage. During the run in phase, the intervention and the final adjustment, insulin was dosed individually to achieve pre-prandial blood glucose levels $<150 \mathrm{mg} / \mathrm{dl}$.

Assuming a significance threshold of 0.025 we calculated that a sample size of 15 study participants would give us a $90 \%$ power to detect a minimum difference of 37 IU insulin dose per day between the diets, a difference that we observed in our pilot study [10]. However, due to the metabolic optimization conducted during the first hospital stay the difference in insulin requirement might have had been lower in the second phase of the crossover design. Therefore, we assumed a power of $80 \%$ to detect an estimated difference of 26 IE insulin per day for 15 study participants at a significance threshold of 0.05 .

Blood samples were collected after an overnight fast on the first, second, third and fourth day of each inpatient stay. Data of preprandial blood glucose self-monitoring were used for the analysis. HbA1c was measured by Bio-Rad's Micromat ${ }^{\mathrm{TM}}$ II (Bio-Rad, Munich, Germany). Blood lipids and other laboratory parameters were measured in the routine laboratory. All values are given as mean \pm SD or median $\left(25^{\text {th }}\right.$ to $75^{\text {th }}$ percentile). For statistical analysis the difference in insulin doses and in laboratory parameters between the third and fourth day and the second day of each inpatient stay was calculated and the effect compared between both phases by using the paired t-test or the Wilcoxon test, as appropriate. In an alternative analysis as proposed by Wellek \& Blettner [11], treatment effects for the first and second inpatient stay of each study participant were calculated and the delta of the second inpatient stay was subtracted from the delta from the first inpatient stay. The resulting effect estimates were then compared between both groups (oatmeal and then control diet vs control diet and then 
- Table 2 Parameters of glucose metabolism during intervention and control inpatient stay expressed as mean values.

\begin{tabular}{|c|c|c|c|c|c|c|}
\hline & \multicolumn{3}{|c|}{ Intervention } & \multicolumn{3}{|c|}{ Control } \\
\hline & \multirow{2}{*}{$\begin{array}{c}\text { Before } \\
\text { Day } 2\end{array}$} & \multicolumn{2}{|c|}{ After } & \multirow{2}{*}{$\begin{array}{c}\text { Before } \\
\text { Day } 2\end{array}$} & \multicolumn{2}{|c|}{ After } \\
\hline & & Day 3 & Day 4 & & Day 3 & Day 4 \\
\hline $\begin{array}{l}\text { Mean glucose } \\
(\mathrm{mg} / \mathrm{dl})\end{array}$ & $141 \pm 45.5$ & $142 \pm 39.5$ & $134 \pm 35.5$ & $152 \pm 36.8$ & $145 \pm 42.7$ & $139 \pm 36.2$ \\
\hline $\begin{array}{l}\text { Fasting glucose } \\
(\mathrm{mg} / \mathrm{dl})\end{array}$ & $173 \pm 72.7$ & & $146 \pm 39.6$ & $143 \pm 41.7$ & & $140 \pm 46.2$ \\
\hline Total insulin (IU) & $112 \pm 36.2$ & $82.0 \pm 30.3$ & $69.9 \pm 29.9$ & $108 \pm 35.0$ & $108 \pm 35.0$ & $102 \pm 37.2$ \\
\hline
\end{tabular}

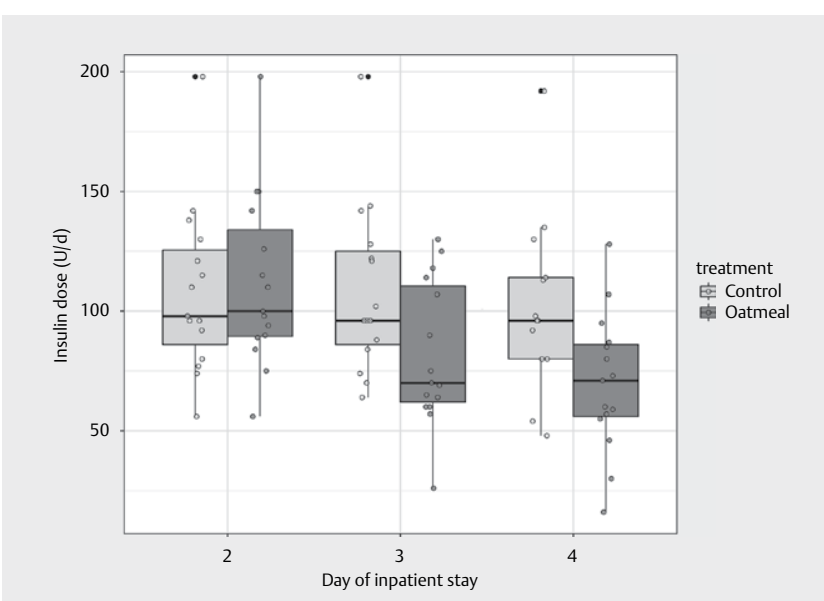

-Fig. 2 Boxplot of insulin dosage during the inpatient stays. Oatmeal treatment was done on day 3 and 4 of the intervention phase while the normal diabetes adapted diet was continued in the control phase. The small black dots represent outliers. Individual data points are shown as filled circles.

oatmeal) by Mann-Whitney $U$ test. A p-value of $<0.05$ was considered to be statistically significant. All analyses were performed using IBM SPSS (version 24) and R (version 3.4.3) statistical software (http://www.r-project.org) [12].

\section{Results}

The mean age of our patients was 58.6(10.1) years and 8 out of 15 were men ( $>$ Table 1 ). Diabetes duration ranged from 3 to 45 years with a median of 10 years. All patients suffered from uncontrolled diabetes, $80.0 \%$ had microvascular and $27.3 \%$ had macrovascular complications.

After two days of diabetes-adapted diet mean blood glucose was 155 (126-172) mg/dl at an insulin dose of 103 (77-134) $U$ at the first inpatient stay and $142(114-164) \mathrm{mg} / \mathrm{dl}$ at an insulin dose of 92.7 (76.7-149) $U$ at the second inpatient stay (Supplementary Table 1S). The only significant difference was a slightly lower triglyceride concentration at the second inpatient stay.

Mean glucose levels and mean insulin dosage during the two inpatient stays are shown in $>$ Table 2 . During the oatmeal intervention, the required insulin dose to achieve a comparable metabolic control was significantly lower as compared to the diabetes mellitus-adapted diet alone. $>$ Fig. $\mathbf{2}$ and $>$ Table $\mathbf{3}$ show the differences in required daily insulin doses and glucose between the days 3 or 4 versus day 2 of the intervention phase and the control phase. While the required insulin dose was significantly reduced following the oatmeal on days 3 and 4 of the intervention phase, there was, as expected, no reduction in the control phase. Despite the marked reduction in insulin dose requirement, there was no significant difference in mean blood glucose levels or fasting glucose levels, neither on the oatmeal phase nor in the control phase. As the first blood draw on day 3 of the intervention phase occurred before oatmeal treatment, no comparison was made for fasting glucose levels on day 3 versus day 2 . On day 4 of the intervention phase, there was a trend towards reduced fasting glucose. The beneficial effect of the oatmeal treatment is further supported by measurement of $\mathrm{HbA} 1 \mathrm{c}$ during the inpatients stays and ambulatory visits 4 to 12 weeks after each hospital stay ( $>$ Table 4). The reduction in $\mathrm{HbA} 1 \mathrm{c}$ was higher following the intervention phase as compared to the control phase, although the difference was only significant at the first ambulatory visit four weeks after the inpatient stay. We also examined the changes in blood lipids, C-peptide, proinsulin, liver enzymes, C-reactive protein and uric acids and found no significant differences between the oatmeal phase and the control phase except for proinsulin (Supplementary Table 2S). Proinsulin increased after oatmeal treatment whereas there was a decreased in the control phase.

We also analysed the results of our crossover trial following the recommendation by Wellek \& Blettner. We calculated the treatment effects of days 3 and 4 versus day 2 for the first and the second inpatient stay of each study participant and then subtracted the effect during the second inpatient stay from the effect of the first inpatient stay. In the case of total insulin requirement, the first study arm (first control, then intervention) showed no insulin dose reduction during the first (control) inpatient stay but a significant reduction during the second (intervention) stay. The negative value from the second stay was then subtracted from the value from the first stay resulting in a positive number and vice versa for the second study arm. The values obtained for both study arms were then compared by a Mann-Whitney U test ( $\triangleright$ Fig. 3). Results were basically the same as for the paired tests. While there was no significant difference in glucose levels the required insulin dose was markedly reduced after oatmeal.

\section{Discussion}

The key results of our crossover study in patients with uncontrolled diabetes mellitus are: (i) two days of oatmeal intervention led to significant reductions in the required daily insulin dose, (ii) mean blood glucose levels and fasting blood glucose levels stayed similar in both groups and (iii) improvements in glucose metabolism in 
> Table 3 Changes in glucose and insulin requirement during intervention and control inpatient stay.

\begin{tabular}{|c|c|c|c|c|}
\hline & Intervention / oatmeal phase & Control phase & P paired t-test & p Wilcoxon test \\
\hline \multicolumn{5}{|l|}{ Days 3 vs Day 2} \\
\hline$\Delta$ mean glucose $(\mathrm{mg} / \mathrm{dl})$ & $1.50 \pm 38.0$ & $-6.64 \pm 28.8$ & 0.374 & 0.307 \\
\hline$\Delta$ fasting glucose $(\mathrm{mg} / \mathrm{dl})$ & - & - & - & - \\
\hline$\Delta$ total insulin (IU) & $-29.8 \pm 19.7$ & $0.133 \pm 10.8$ & 0.000143 & 0.017 \\
\hline$\Delta$ mean glucose + total insulin & $-28.3 \pm 48.0$ & $-6.51 \pm 31.9$ & 0.036 & 0.041 \\
\hline \multicolumn{5}{|l|}{ Days 4 vs Day 2} \\
\hline$\Delta$ mean glucose $(\mathrm{mg} / \mathrm{dl})$ & $-6.88 \pm 29.2$ & $-12.8 \pm 31.5$ & 0.394 & 0.363 \\
\hline$\Delta$ fasting glucose $(\mathrm{mg} / \mathrm{dl})$ & $-30.29 \pm 46.2$ & $-3.07 \pm 43.7$ & 0.102 & 0.069 \\
\hline$\Delta$ total insulin (IU) & $-41.9 \pm 30.1$ & $-1.15 \pm 14.5$ & 0.003 & 0.006 \\
\hline$\Delta$ mean glucose + total insulin & $-48.8 \pm 42.4$ & $-11.4 \pm 41.2$ & 0.004 & 0.008 \\
\hline
\end{tabular}
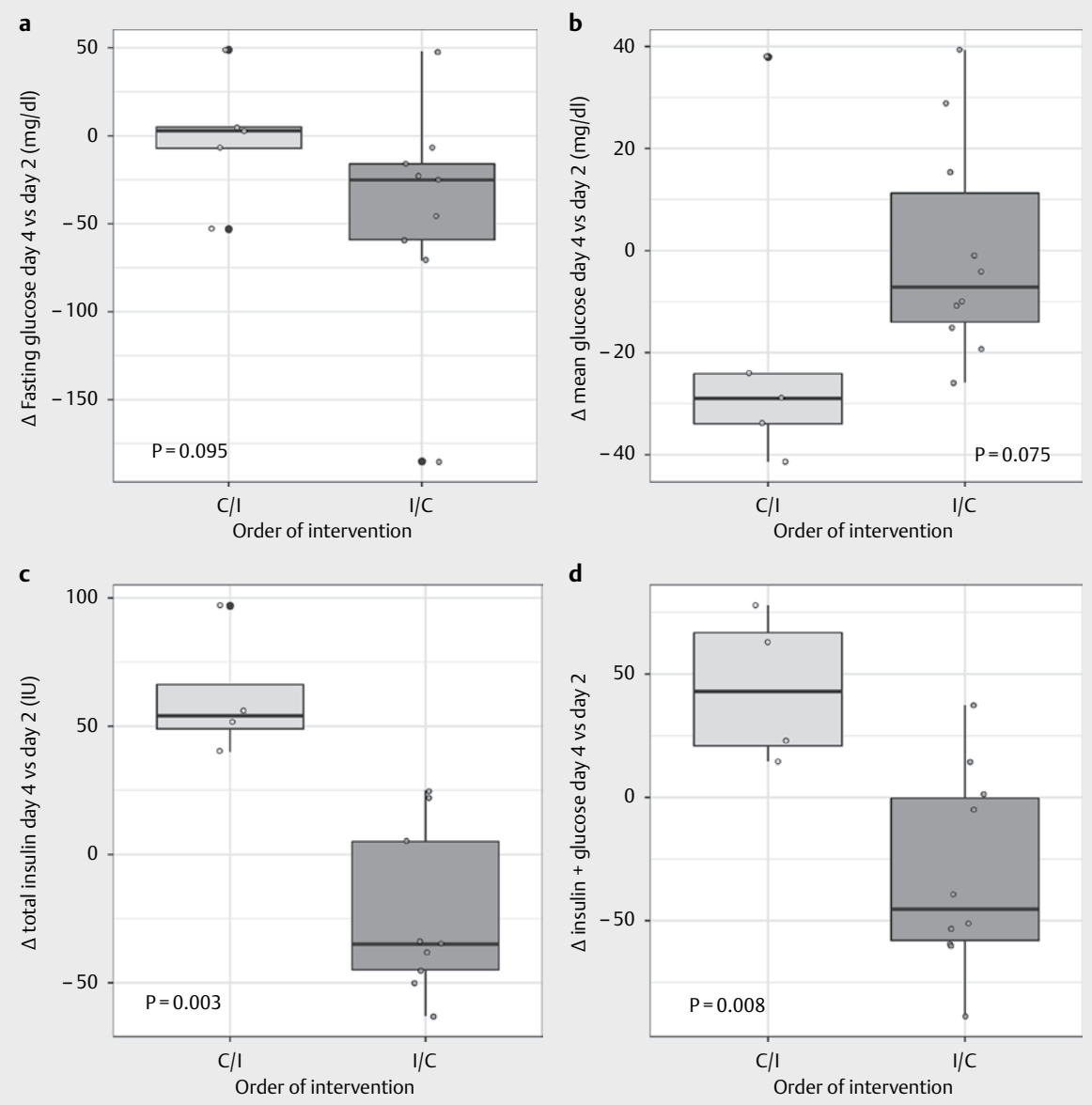

Fig. 3 Comparison of intervention efficiency. The differences in fasting glucose $\mathbf{a}$, mean glucose $\mathbf{b}$, total insulin $\mathbf{c}$ and total insulin + mean glucose concentration $\mathbf{d}$ were calculated separately for each inpatient stay (day 4 minus day 2 ). The $\Delta$ from the second inpatient stay was subtracted from the $\Delta$ from the first inpatient stay and the resulting values are shown as boxplots, separately for both arms of the trial. A Mann-Whitney-U test was used to compare the effect between both groups. The small black dots represent outliers. Individual data points are shown as filled circles. C/I: control then intervention; $\mathrm{I} / \mathrm{C}$ : intervention then control.

- Table 4 Differences in HbA1c after intervention or control inpatient stay.

\begin{tabular}{|l|c|c|c|}
\hline \multirow{2}{*}{ Time after inpatient stay } & \multicolumn{2}{|c|}{$\Delta$ HbA1c (\%) } & \\
\cline { 2 - 4 } & Intervention & Control & p t-test \\
\hline Four weeks & $-1.06 \pm 1.33$ & $-0.22 \pm 0.71$ & 0.020 \\
\hline Eight weeks & $-0.79 \pm 1.23$ & $-0.48 \pm 1.04$ & 0.526 \\
\hline Twelve weeks & $-0.67 \pm 0.78$ & $-0.30 \pm 1.34$ & 0.032 \\
\hline
\end{tabular}


terms of reduced $\mathrm{HbA} 1 \mathrm{c}$ were detectable even four weeks after the oatmeal intervention.

These results confirm the results of our uncontrolled pilot study [10]. Mean reduction in insulin dosage following oatmeal treatment was $37 \%(112 \pm 36.2 \mathrm{U} / \mathrm{d}$ before vs $69.9 \pm 29.9 \mathrm{U} / \mathrm{d}$ after two days of oatmeal) in OMA-IR while there had been a reduction of $42.5 \%$ in the pilot study. The effect in this trial is presumable slightly lower because the current study compared two hypocaloric diets with similar caloric restrictions whereas in the pilot study caloric uptake was different between the intervention and control group.

How could this short intervention lead to such dramatic results with still a decreased HbA1c one month later? There are some potential explanations. First, the intervention was rigidly hypocaloric with regard to the BMI of the patients. Lim et al. demonstrated normalisation of beta cell function and hepatic insulin sensitivity with an eight weeks formula diet with only $600 \mathrm{kcal}$ daily in eleven patients with diabetes mellitus [13]. The hepatic insulin resistance was already broken within the first week of calorie restriction. For the normalization of the beta cell function the triglyceride content of the pancreas (as determined by MRT) had to be normalized, which needed a further 7 weeks. As a proof of concept, the DIRECT trial from the same group demonstrated that a hypocaloric formula diet for 3-5 months resulted in a $46 \%$ remission rate of diabetes mellitus over one year [14].

However, caloric restriction may not explain the striking differences seen between our two intervention groups. Such an effect may be explained by increased ingestion of beta-glucans along with the oats. There are a number of interventional trials, which demonstrate reduction of postprandial glycaemic excursions with beta glucans [15] and a recent trial confirmed a dose-response relationship [16]. Trials with intact grain demonstrated even more pronounced results. Therefore, part of the reduced glycaemic excursions may be a result of the intake of beta-glucans and fibre within the oatmeal [9].

Lastly, we hypothesize that short chain fatty acids (SCFA) resulting from the fermentation of fibre within the colon may modulate satiety and motility of the intestinal tract. Zhao et al. demonstrated that a fibre-enriched diet transforms the gut microbiome and leads to SCFA production [17]. They selected 15 bacterial strains by a fibre enriched diet that were correlated with metabolic improvement and verified this hypothesis in murine models.

In summary, the combination of caloric restriction, reduction of glycaemic load and the production of SCFA may explain our findings of a rapid improvement of insulin resistance in our cohort.

\section{Limitations}

Due to the design of the study, we were only able to control the patients within the hospital. For almost all patients within this trial the oatmeal intervention was the first time to get an idea of self-efficacy. We advised our patients not to eat any oatmeal during the outpatient follow-up but we did not perform a structural evaluation of this possible bias. However, considering the simplicity of this diet, we cannot exclude that patients have performed some outpatient oatmeal days. The cinnamon used for seasoning of oatmeals may itself have a small insulinotropic effect. However, the amount is not comparable to the doses used in studies that reported such effects $[18,19]$.A possible limitation of this study could be that the allocation of the study arms was done by flipping a coin, which resulted in unequal group sizes. More patients received the oatmeal intervention at the first inpatient stay. However, this fact does not severely impair the crossover design due to the long period between the interventions. Another limitation may be that insulin requirement was estimated clinically by keeping pre-prandial blood glucose levels $<150 \mathrm{mg} / \mathrm{dl}$ rather than in a euglycemic clamp experiment.

\section{Conclusion}

In this crossover study, a two-day oatmeal intervention in patients with uncontrolled T2DM allowed for a highly significant reduction of required daily insulin dose while maintaining adequate metabolic control as compared to diabetes adapted diet only. The beneficial effects of the intervention might last for several weeks as shown by the lower HbA1c four weeks after the intervention phase.

\section{Acknowledgements}

The authors extend appreciation to the participants our study "Carbohydrate Days as Simple and Efficient Therapy for Patients with Type 2 Diabetes Mellitus and Insulin Resistance: Oatmeal and Insulin Resistance (OMA-IR)"; without their collaboration this article would not have been written.

\section{Conflict of Interest}

No conflict of interest has been declared by the authors.

\section{References}

[1] von Noorden C. Hafercuren bei schwerem diabetes mellitus. Klin Wochenschr 1903; 36: 817-821

[2] Allen FM. Starvation diet for diabetic patients. Am J Med Sci 1915; 150: $480-450$

[3] Mazur A. Why were "starvation diets" promoted for diabetes in the pre-insulin period? Nutr J 2011; 10: 23

[4] Herrick JB. The oatmeal diet in the treatment of diabetes mellitus. JAMA 1908; L: 861-865

[5] Oyen D, Chantelau EA, Berger M. Die Entwicklung der Diabetesdiät seit 1950. In: Zur Geschichte der Diabetesdiät. Berlin Heidelberg: Springer-Verlag Berlin Heidelberg New York Tokyo; 1985

[6] Zerm R, Kröz M. Hafertage bei diabetes mellitus typ 2 und ausgeprägter Insulinresistenz. Ernährung \& Medizin 2014; 29: 13-15

[7] Beta-glucans from oats and barley related health claims. EFSA Journal 2011; 9: 2207

[8] Hou Q, Li Y, Li L et al. The metabolic effects of oats intake in patients with type 2 diabetes: A systematic review and meta-analysis. Nutrients 2015; 7: 10369-10387

[9] Weickert MO, Pfeiffer AF. Metabolic effects of dietary fiber consumption and prevention of diabetes. J Nutr 2008; 138: 439-442

[10] Lammert A, Kratzsch J, Selhorst ] et al. Clinical benefit of a short term dietary oatmeal intervention in patients with type 2 diabetes and 
severe insulin resistance: A pilot study. Exp Clin Endocrinol Diabetes 2008; 116: 132-134

[11] Wellek S, Blettner M. On the proper use of the crossover design in clinical trials: Part 18 of a series on evaluation of scientific publications. Dtsch Arztebl Int 2012; 109: 276-281

[12] R Core Team. R: A language and environment for statistical computing.Vienna, Austria: R Foundation for Statistical Computing; 2018 URL https://www.R-project.org/

[13] Lim EL, Hollingsworth KG, Aribisala BS et al. Reversal of type 2 diabetes: Normalisation of beta cell function in association with decreased pancreas and liver triacylglycerol. Diabetologia 2011; 54: 2506-2514

[14] Lean ME, Leslie WS, Barnes AC et al. Primary care-led weight management for remission of type 2 diabetes (DiRECT): An open-label, cluster-randomised trial. Lancet 2018; 391: 541-551

[15] Tosh SM. Review of human studies investigating the post-prandial blood-glucose lowering ability of oat and barley food products. Eur ] Clin Nutr 2013; 67: 310-317
[16] Wolever TMS, Jenkins AL, Prudence $K$ et al. Effect of adding oat bran to instant oatmeal on glycaemic response in humans - a study to establish the minimum effective dose of oat beta-glucan. Food Funct 2018; 9: 1692-1700

[17] Zhao L, Zhang F, Ding X et al. Gut bacteria selectively promoted by dietary fibers alleviate type 2 diabetes. Science 2018; 359: 1151-1156

[18] Allen RW, Schwartzman E, Baker WL et al. Cinnamon use in type 2 diabetes: an updated systematic review and meta-analysis. Ann Fam Med 2013

[19] Zare R, Nadjarzadeh A, Zarshenas MM et al. Efficacy of cinnamon in patients with type II diabetes mellitus: A randomized controlled clinical trial. Clin Nutr 2018, doi:10.1016/j.clnu.2018.03.003 